\title{
Human lymph node morphology as a function of age and site*
}

\author{
P LUSCIETI, $\dagger$ Th HUBSCHMID, H COTTIER, MW HESS, AND LH SOBIN \\ From the Institute of Pathology, University of Bern, Bern, and the $\ddagger$ Cancer Unit, World Health \\ Organization, Geneva, Switzerland
}

SUMMARY Cervical, axillary, cubital, inguinal, popliteal, and mesenteric lymph nodes from subjects of various ages who had died a sudden death were examined histologically. Care was taken to establish by morphometry the proportional distribution in lymph node cross-sections of cortical, paracortical, and medullary areas. In addition, numbers and surface areas of cross-sectioned germinal centres were registered. Important differences related to age and anatomical site of lymph nodes were established by this survey. Germinal centre formation, particularly evident in infants and children, less so in young adults, and often absent in ageing individuals, was most impressive in lymph nodes normally exposed to antigenic stimulation (mesenteric and cervical lymph nodes). Paracortical and medullary areas exhibited a slight but gradual reduction with advancing age. Replacement of lymphatic parenchyma by fat tissue (lipomatous atrophy) was a characteristic of more peripheral lymph nodes usually subjected to little antigenic stimulation, that is, cubital, axillary, and popliteal nodes. It should be emphasised that both age-related and regional differences have to be taken into account in a meaningful functional interpretation of lymph node morphology.

The lymph node system constitutes the major part of the peripheral lymphoid tissues in the mammalian organism. Because they drain anatomically distinct tissue areas, these structures often represent the primary sites of interaction between antigenic material and immunologically competent cells. Hence, structural changes in lymph node morphology directly reflect local immune reactivity.

The possibility that information on immunological function may be gained from registering changes in lymph node architecture and cellularity ${ }^{1}$ has both theoretical and practical implications. For instance, correlation of lymphoid tissue morphology with other parameters of immune responsiveness in animal experiments greatly enhanced our knowledge of age-related differential changes in $T$ and $B$ cell-mediated immunity. ${ }^{2}$ Associations between lymph node morphology and prognosis have been demonstrated for cancers of lung, cervix, colon, and breast. ${ }^{3-6}$

It is of practical importance that in the diagnostic

*Supported by the Swiss National Science Foundation †Present address: Istituto Cantonale di Patologia, 6600 Locarno, Switzerland

Received for publication 25 June 1979 evaluation of lymph node sections both localisation of the node and the age of the patient be taken into account. As an example, the prominence of germinal centres in a cubital lymph node clearly indicates an ongoing antigenic stimulation by-possibly infectious-material present in corresponding tissues, while germinal centres are normally present in tonsils, gut-associated lymphoid tissues, and mesenteric lymph nodes at least of younger individuals.

Systemic studies of lymph node morphology as a function of both age and lymph node location in normal human subjects would provide the necessary background for meaningful functional interpretation of tissue sections. Since such information is scarce at present, this survey was undertaken. It was aimed at providing insight into the 'normal' histology of six representative human lymph node sites in seven different age groups.

\section{Tissue specimens and methods}

LYMPH NODES

Most lymph nodes were collected during necropsy of cases of sudden death (accidents, coronary heart disease, etc.); a small number of nodes was submitted as biopsy specimens. Care was taken to exclude from the study all cases with clinical or histo- 
logical evidence of neoplastic and/or infectious disease. According to the age of the individual at the time of lymph node removal (necropsy or biopsy), the following age groups were considered: 'premature' (up to 1 month before term), 'newborns' ( 1 to 10 days after birth), 'infants' ( 1 to 12 months), 'children' ( 3 to 10 years), 'young adults' (20 to 30 years), 'adults' (40 to 60 years), and 'the elderly' (70 to 90 years). Lymph node sites examined included cervical (CeLN), axillary (ALN), cubital (CuLN), inguinal (ILN), popliteal (PLN), and mesenteric (MLN) locations.

The numbers of individual lymph nodes evaluated in the course of this study are listed in the Table for each age group.

Table Number of lymph nodes included in study per site and age group

\begin{tabular}{|c|c|c|c|c|c|c|c|}
\hline \multirow[t]{2}{*}{ Age group } & & \multicolumn{6}{|c|}{ Lymph node site* } \\
\hline & & CeLN & $A L N$ & $C u L N$ & $I L N$ & $P L N$ & $M L N$ \\
\hline Premature & $\dagger$ & 4 & 3 & 2 & 3 & 1 & 3 \\
\hline Newborn & $(1-10 d)$ & 5 & 4 & 4 & 6 & 4 & 6 \\
\hline Infants & (1-12 mth) & 4 & 3 & - & 3 & 1 & 4 \\
\hline Children & (3-10 yr) & 8 & 6 & 2 & 8 & 6 & 9 \\
\hline Young adults & $(20-30 \mathrm{yr})$ & 8 & 13 & 3 & 10 & 5 & 12 \\
\hline Adults & $(40-60 \mathrm{yr})$ & 4 & 3 & 2 & 5 & 1 & 3 \\
\hline Elderly & $(70-90 \mathrm{yr})$ & 20 & 19 & 2 & 15 & 3 & 16 \\
\hline
\end{tabular}

*For abbreviations see 'Tissue specimens and methods'.

†Up to 1 month before term.
TISSUE PROCESSING

The lymph nodes were fixed in $4 \%$ formalin, buffered at $\mathrm{pH} 7 \cdot 4$; before embedding in paraplast, the fixed nodes were carefully halved with a razor blade in a plane perpendicular to their longer diameter. Cross-sections $(4 \mu \mathrm{m})$ were stained with haemalumeosin.

\section{PLANIMETRY}

Microscopic pictures of each lymph node crosssection were projected on stiff paper. Cortical, paracortical, and medullary zones were identified, based on criteria established previously ${ }^{1}$ and marked. Individual zones were cut out and weighed; surface areas (expressed in $\mathrm{mm}^{2}$ ) were determined from weight by simple calculation. Germinal centres were enumerated and registered per $\mathrm{cm}^{2}$. Mean values $( \pm \mathrm{SD})$ are given for all figures.

\section{Results}

Age-dependent changes in the relative size of the follicular (outer) cortex per cross-sectioned lymph node are presented in Figure 1. Values ranged from between 5 and $10 \%$ in prematures and newborns for all nodes; they were highest in MLN, CeLN and, perhaps, PLN. Maximal development of this lymph node area may be noted during the first year of life: in this group, cortical deployment was significantly

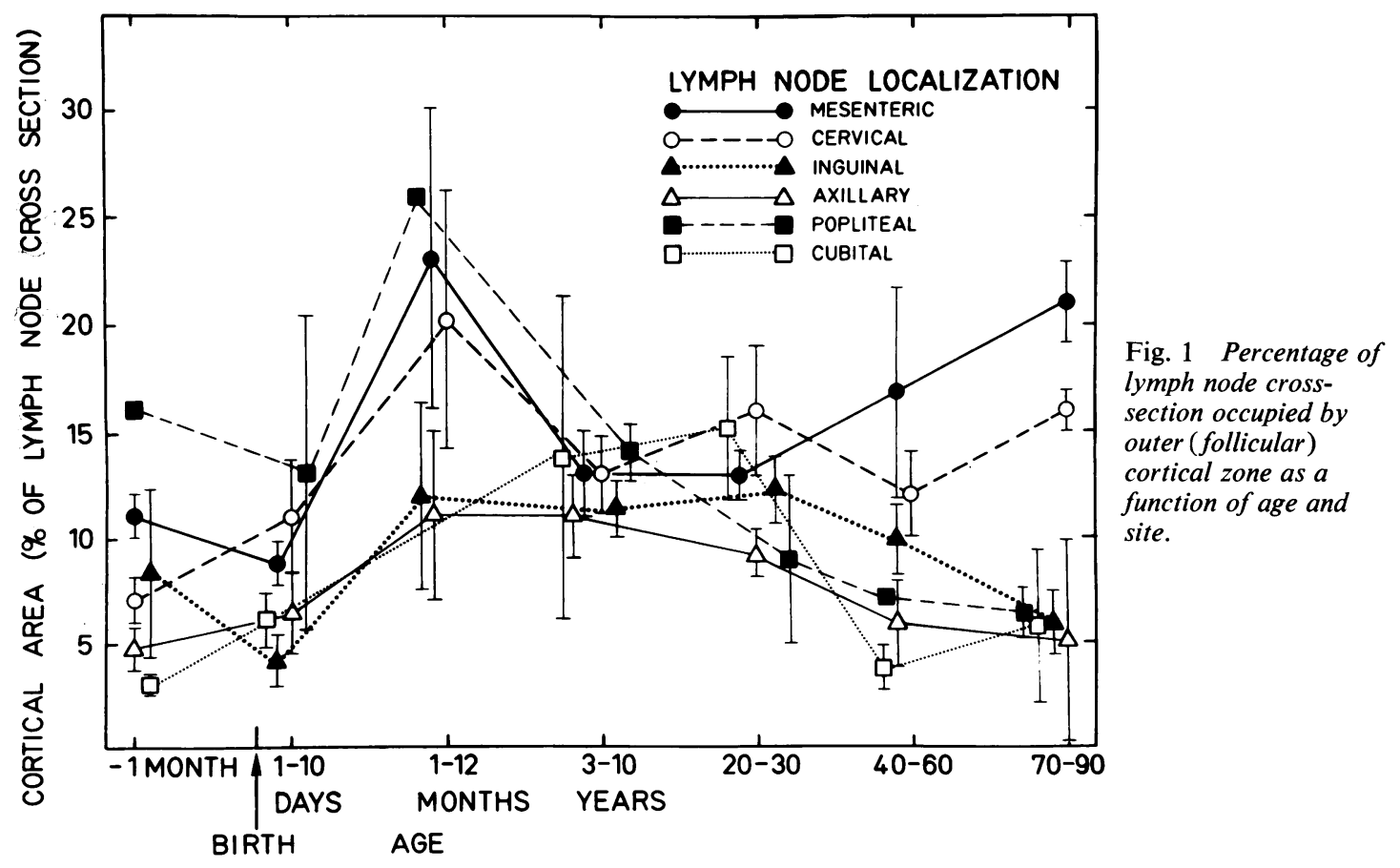


greater in MLN and CeLN (means of $20 \%$ ) than in other nodes. This pattern largely reflects total germinal centre volumes as seen in Figure 3 . The value registered for PLN is based on a single measurement and, thus, may not be representative. In infants, children, and young adults, the follicular cortex occupied between 10 and $25 \%$ of lymph node crosssections. This proportion declined gradually with advancing age to values corresponding to those of the newborn. Again, a divergent behaviour was registered for MLN and CeLN as compared to that of other lymph nodes: the cortex of the former exhibited a relative re-expansion to 20 and $15 \%$, respectively, in very old age. As documented in Fig. 2, this latter change was due not only to renewed germinal centre formation but rather to a concomitant expansion of so-called primary follicles.

Germinal centres were absent in lymph nodes of the examined premature and newborn infants (Fig. 2). These structures appeared during the first year, and the highest numbers were found in this age group, with the following exceptions: germinal centre development in ILN was most pronounced in children and practically absent in CuLN. Mean numbers of germinal centres were highest in MLN, CeLN, and ALN, in that order; the difference between values of the last two nodes is statistically significant. However, when germinal centre areas are considered (Fig. 3), it is evident that a difference between MLN and CLN does not exist, and that these structures were considerably larger in MLN and CeLN than in all other lymph nodes.

With regard to the medullary zone (Fig. 4), a tendency was noted to maintain approximate values of $40 \%$ throughout life, after somewhat higher percentages around birth. Notable exceptions are presented by CeLN, in which a more pronounced reduction was registered in adults and the elderly.

In most lymph nodes, the relative size of the paracortical (diffuse cortical) areas was maximal around birth and showed a very slow, but gradual decrease throughout life with minor regional differences (Fig. 5). The CeLN exhibited a completely different behaviour in that the paracortical zone was minimally developed before and after birth, reached levels comparable to those of other lymph nodes in infants and children, but continued to expand in older individuals.

The extent of replacement of lymph node parenchyma by fat tissue (lipomatous atrophy) in lymph nodes of different localisations is shown in Figure 6. While MLNs, CeLNs, and ILNs showed minimal signs of lipomatosis in all age groups, CuLNs, ALNs, and PLNs to a certain extent were already lipomatous during infancy and childhood; lipotamous changes increased with advancing age in

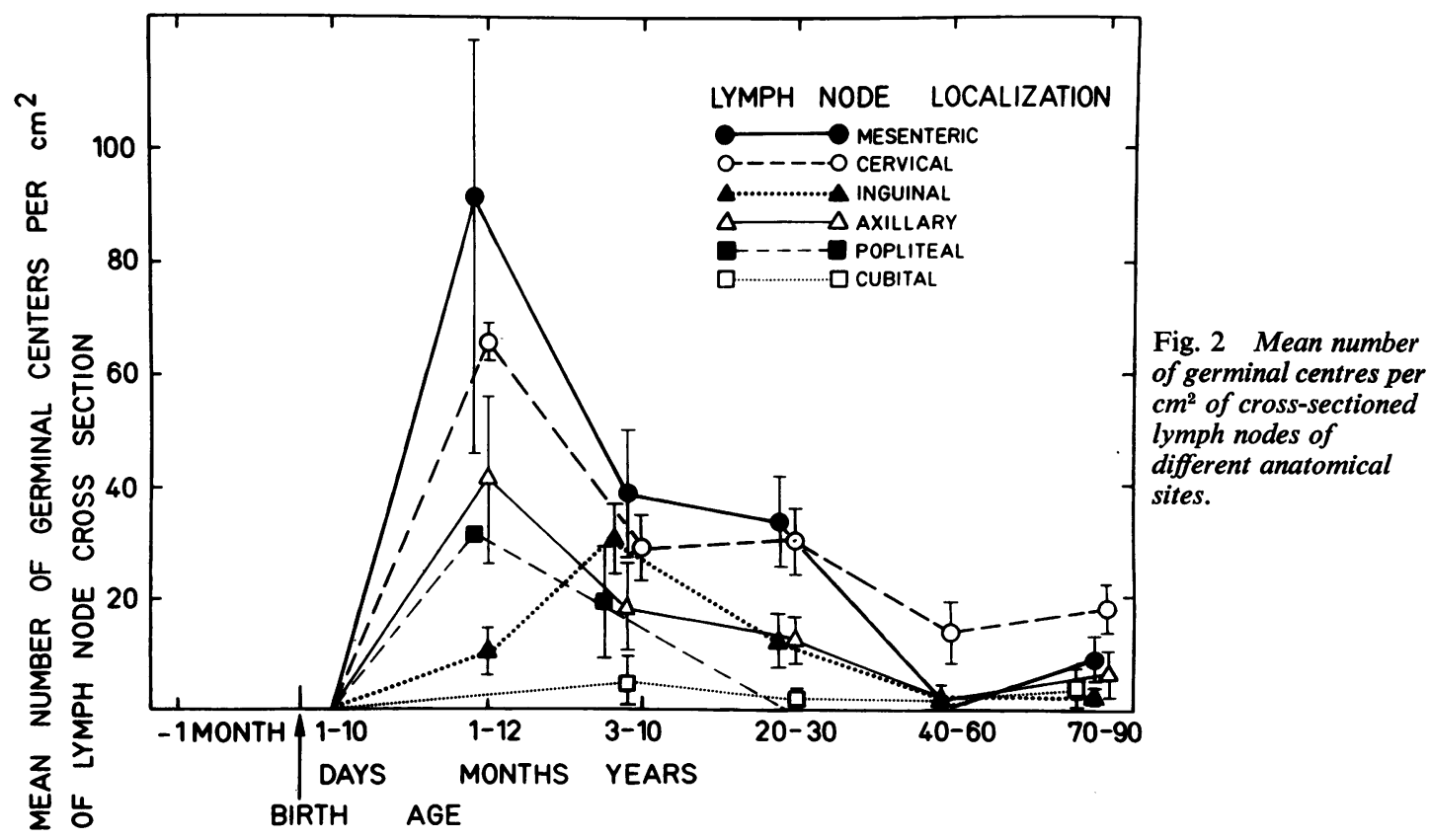




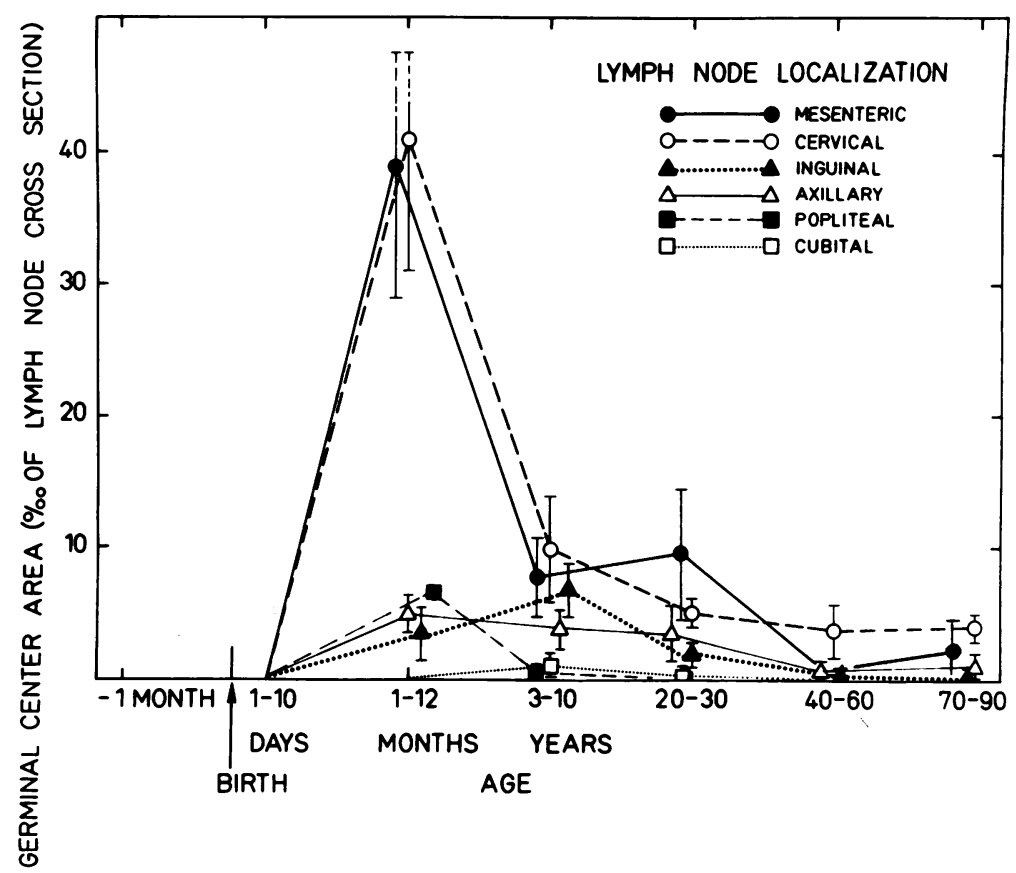

Fig. 3 Percentage of lymph node cross-section occupied by germinal centres as a function of age.

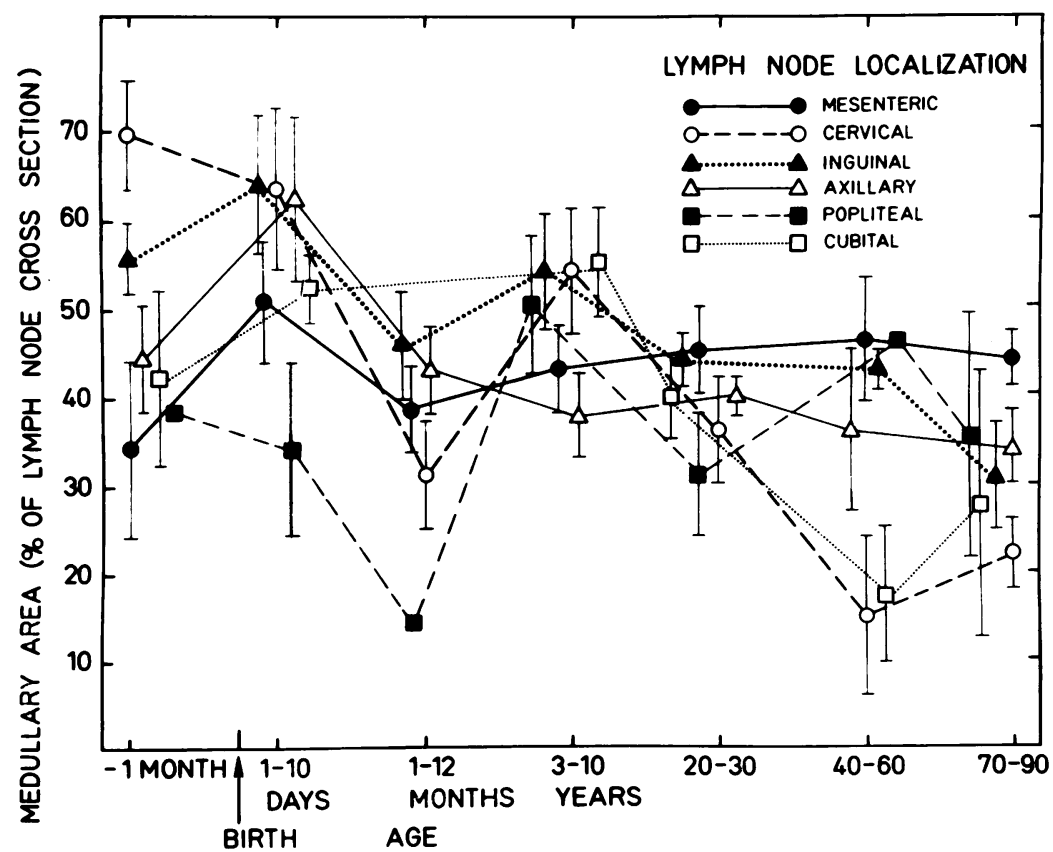

Fig. 4 Percentage of lymph node cross-section occupied by medullary zone as a function of age. 


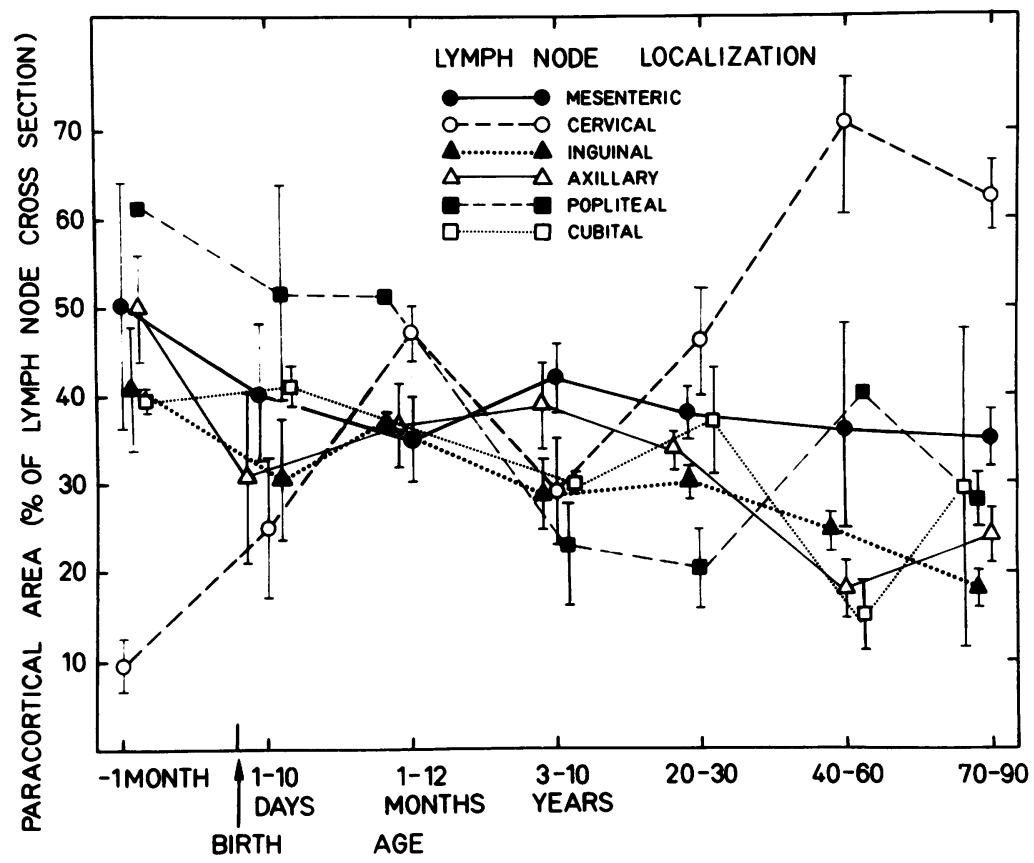

Fig. 5 Percentage of lymph node cross-section occupied by paracortical zone (deep or diffuse cortex) as a function of age.

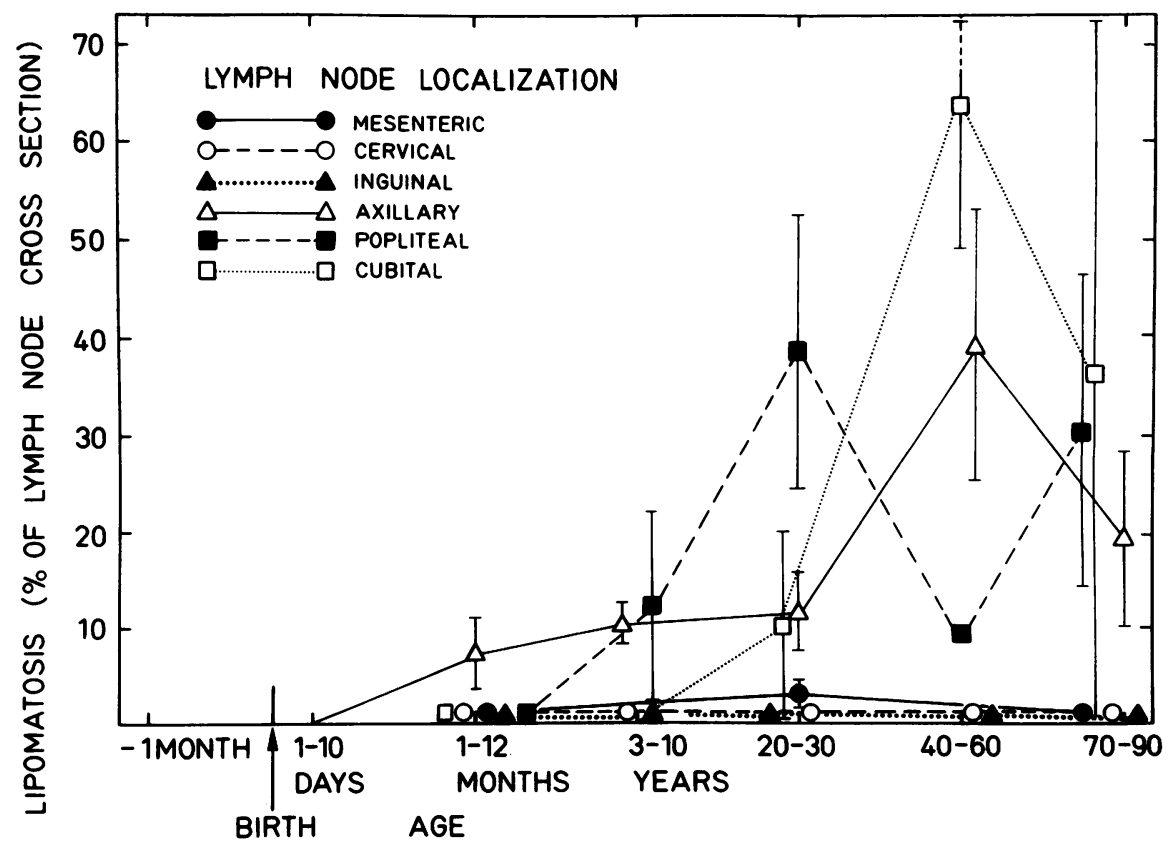

Fig. 6 Relative extent of lymph node lipomatosis (\% of lymph node cross-section) as a function of age. 
these lymph nodes and reached considerable levels; for example, the cut surface of the average $\mathrm{CuLN}$ in adults consisted of more than $60 \%$ fat tissue.

\section{Discussion}

Both development and partial decline of immune capacities during the life of mammalian organisms appear to be the consequence of a complex interplay of various factors. Among these, attention has been focused in experimental and clinical studies on changes in function of immunologically competent cells or shifts in relative proportions of lymphocyte subpopulations as well as on variations in environmental influences. ${ }^{2}$ Based on experimental evidence obtained, for example, from transfusing lymphocytes of young donors into irradiated elderly recipients and vice versa, approximately $90 \%$ of the age-related impairment of immune functions were estimated to be due to intrinsic changes in the immunocompetent cell systems ${ }^{7}$ while the host's microenvironmentin its broadest sense-was held responsible for the rest. ${ }^{8}$ These estimates have to be taken as approximations since mutual influences of intrinsic and extrinsic factors in vivo, for example, changes of migratory behaviour of cells by environmental agents, are difficult to assess.

It is generally believed that the age-dependent changes of the lymphoid system, that is, expansion in the postnatal period and gradual reduction after childhood, are largely influenced by thymic functions. Recent reports indicate that the number of circulating lymphocytes in humans decreases progressively during and after middle age, and by the sixth decade a level is attained that corresponds to approximately $70 \%$ of that observed in young adults. This decline in blood lymphocyte numbers is usually due to a reduction of the circulating $T$ cell mass. ${ }^{9}$ Exceptions to this rule are, however, quite numerous. ${ }^{10}$ Furthermore, a decrease in the number of cells bearing $\mathrm{T}$ lymphocyte membrane markers does not necessarily mean that the number of thymus-derived cells has fallen because a fraction of $T$ cells could lack the usual characteristics due to incomplete differentiation or other mechanisms. Finally, it should be emphasised that the number of circulating lymphocytes does not always reflect the mass of these cells located in lymphoid tissues. It is, therefore, of interest to note that, in our series, the relative size of the paracortical area in four out of six lymph node sites showed a gradual decline from early childhood to advanced age, a finding that would support the above reports on an age-dependent involution of the $T$ cell system. However, this pattern was not seen in all lymph node locations. In particular, cervical lymph nodes-many of which drain the lymphoepithelial structures in the pharynx, particularly the tonsils-exhibit the contrary, that is, an increase in relative volume up to the age of at least 40-60 years. We suggest from this observation that the involutional process regarding tissue $T$ cell mass is not the same in all lymph nodes, but rather shows substantial regional differences or even a reverse behaviour. It is reasonable to assume that the blood $\mathrm{T}$ lymphocyte numbers reflect to some extent a mixture of cells having resided in, or passed through, lymph nodes of different areas, so that only a slight to moderate reduction in total $\mathrm{T}$ cell mass would result with advancing age (see Fig. 5).

In discussing $\mathrm{T}$ cell numbers, one should bear in mind that they do not necessarily go in parallel with thymic functions in general. We have to recognise the possibility that certain subpopulations of thymusderived lymphocytes exhibiting different activities may have changed with advancing age. In fact, several reports mention an age-dependent decline in $\mathrm{T}$ cell helper functions; others raise the possibility that the higher incidence of autoimmune disease in elderly people as compared to younger individuals could be due to decreased suppressor functions of $T$ cells with advancing age. ${ }^{1011}$ It is too early to draw definitive conclusions in this respect from the available information. Tests in vitro appear to indicate that the $T$ cell responsiveness to stimuli such as new antigens or $\mathrm{T}$ cell mitogens may be reduced in older persons. If these reports should prove to be of general validity, it could mean that not only the numbers of $T$ cells of a given category but also the functional capacity of the individual lymphocyte may to some extent deteriorate with age, as has been shown for many other cell types. ${ }^{12}$

Assuming that the follicular cortex contains more than $90 \%$ B cells, ${ }^{13}$ the present data indicate that an age-dependent reduction of the $B$ cell mass in this location was evident from childhood to senescence in all examined lymph node sites, except mesenteric and-to some extent-cervical lymph nodes. In other words, regional differences with regard to the involutionary pattern were also observed with regard to this classical B cell area. Again, the numbers of blood B lymphocytes which seem to change little with age ${ }^{9}$ do not reflect the situation in all lymph nodes. They give no insight into the more sessile compartments of B cells which, as demonstrated here, exhibit marked regional differences. On the whole, B cell responsiveness to stimulation with at least certain antigens seems to decrease markedly with age. Studies on young and ageing mice indicated that this decline was due to $(a)$ a decrease with age in the numbers of antigen-sensitive immunocompetent units, and (b) a reduction in immunologic burst size, that is, the number of antibody- 
forming effector cells generated by an immunocompetent unit. ${ }^{7}$ In man, serum IgG and IgA levels increase with age whereas serum IgM tends to decrease. This corresponds to the finding of relatively more plasma cells in lymph nodes of ageing persons as compared to young individuals; the morphological aspect of IgM-producers differs from that of typical plasmacytes. It should be kept in mind that B cell functions, in particular, responses to socalled thymus-dependent antigens, largely rely on $\mathrm{T}$ cell activities. Therefore, these two lymphocyte populations may not be considered as functionally separate systems.

Of all the parameters examined in this study, germinal centre numbers and size showed the most impressive age-dependent changes. These structures, which result from B cell proliferation and appear to serve primarily $B$ cell memory, ${ }^{14}$ are relatively most numerous and attain the greatest volumes in mesenteric and cervical lymph nodes, that is, nodes draining areas with heavy microbial contamination and equipped with lymphoepithelial structures ('immunological contact organs') such as Peyer's patches, the appendix, intestinal lymph follicles, and pharyngeal tonsils. Similar observations were made on other mammalian species, in particular the mouse. ${ }^{15}$ It is noteworthy that the mean number of germinal centres per unit area of lymph node cross-section is highest by far in infants 1 to 12 months of age. The same pertains to the proportion of lymph node crosssections occupied by germinal centres. With advancing age, these structures are found with decreasing frequency. The age-dependent overall decline in germinal centre formation is not necessarily reflecting a deterioration of the individual's capacity to generate B cell memory since it is well recognised that repeated stimulation ('hyperimmunisation') with the same antigen leads to a progressive disappearance ('dissolution') of these discrete foci of lymphoid cell proliferation. In addition, it appears reasonable to assume that fewer and fewer 'new' antigens are encountered with advancing age. In old mice the capacity to respond to primary antigenic stimulation with de novo formation of germinal centres is reduced but not totally eliminated. Finally, it should be stressed that qualitative changes may occur with advacing age, for example, a greater tendency to produce autoimmune antibodies and/or monoclonal immunoglobulins. It was shown recently that anti-Ig-induced proliferation of murine splenic B cells is an age-associated phenomenon rarely seen in young animals. ${ }^{16}$ The biology of ageing is still poorly understood. ${ }^{12} 17$ Thus, a final evaluation of the data presented in this report must await further progress in this field.

For the pathologist concerned with diagnostic evaluation of lymph node sections, ${ }^{18}$ the present data may serve as a guide-line with respect to regional and age-related differences in the histological appearance of the lymphoreticular tissue. For instance, absence of germinal centres in mesenteric lymph nodes of a 1-year-old infant certainly reflects an abnormal state while the same finding in cubital nodes would be normal. Large and/or numerous germinal centres in lymph nodes of elderly persons, on the other hand, most probably signal that a humoral response of unusual dimensions is going on in the respective area.

It is of interest that lipomatous atrophy was essentially restricted to cubital, axillary, and popliteal lymph nodes, that is, to sites which normally exhibit minimal signs of antigenic stimulation. However, it is possible that other factors may be involved in this particular age-dependent change.

\section{References}

${ }^{1}$ Cottier H, Turk J, Sobin L. A proposal for a standardized system of reporting human lymph node morphology in relation to immunological function. J Clin Pathol 1973;26:317-31 (and Bulletin of the World Health Organization 1972;47:375-408).

${ }^{2}$ Yunis EJ, Fernandes G, Good RA. Aging and involution of the immunological apparatus. In: Twomey JJ, Good RA, eds. The Immunopathology of Lymphoreticular Neoplasms (Comprehensive Immunology vol 4). New York-London: Plenum Medical Book Company, 1978:53-80.

${ }^{3}$ Kaufmann M, Wirth K, Scheurer J, Zimmermann A, Luscieti P, Stjernswärd J. Immunomorphological lymph node changes in patients with operable bronchogenic squamous cell carcinoma. Cancer $1977 ; 39: 2371-7$.

${ }^{4}$ Van Nagell JR, Jr, Donaldson ES, Parker JC, Van Dyke AH, Wood EG. The prognostic significance of pelvic lymph node morphology in carcinoma of the uterine cervix. Cancer 1977;39:2624-32.

${ }^{5}$ Pihl E, Malahy MA, Khankhanian N, Hersh EM, Mavligit GM. Immunomorphological features of prognostic significance in Duke's class B colorectal carcinoma. Cancer Res 1977;37:4145-9.

${ }^{6}$ Tsakraklides V, Olson P, Kersey JH, Good RA. Prognostic significance of the regional lymph node histology in cancer of the breast. Cancer 1974;34: 1259-67.

7 Perkins EH, Makinodan T. Nature of humoral immunologic deficiencies of the aged. In: Proceedings of the 1st Rocky Mountains Symposium on Ageing. Colorado: Colorado State University, 1971 :80-103.

${ }^{8}$ Makinodan T, Adler WH. Effects of aging on the differentation and proliferation potentials of cells of the immune system. Fed Proc 1975;34:153-8.

${ }^{9}$ Diaz-Jouanen E, Williams RC, Jr, Strickland RG. Age-related changes in T and B cells. Lancet 1975; 1 :688-9. (Letter.) 
${ }^{10}$ Hallgren HM, Yunis EJ. Suppressor lymphocytes in young and aged humans. J Immunol 1977;118: 2004-8.

${ }^{11}$ Good RA, Yunis EJ. Association of autoimmunity, immunodeficiency and aging in man, rabbits and mice. Fed Proc 1974;33:2040-50.

${ }^{12}$ Finch CE, Hayflick L, eds. Handbook of the Biology of Aging. New York: Van Nostrand Reinhold Company, 1977.

${ }^{13}$ Weissman IL, Gutman GA, Friedberg SH. Tissue localization of lymphoid cells. Series Haematol 1974; 7:482-504.

${ }^{14}$ Grobler P, Bürki H, Cottier H, Hess MW, Stoner RD. Cellular bases for relative radioresistance of the antibody-forming system at advanced stages of the secondary response to tetanus toxoid in mice. $J$ Immunol 1974;112:2154-65.

${ }^{15}$ Schwander R, Aebi S. The post-natal development of peripheral lymph-nodes in mice. II. Changes in lymph-node cellularity. Thesis, University of Berne, Switzerland, 1978.

${ }^{16}$ Weiner HL, Scribner DJ, Moorhead JW. Antiimmunoglobulin stimulation of murine lymphocytes. IV. Re-expression and fate of cell surface receptors during stimulation. J Immunol 1978;120:1907-12.

${ }^{17}$ Martin E, Junod JP. Abrégé de Gérontologie, 2nd ed. Paris: Masson. Berne: H Huber, 1977.

${ }^{18}$ Lennert K, ed. Lymphknoten: Diagnostik in Schnitt und Ausstrich. Cytologie und Lymphadenitis. (Handbuch der Speziellen Pathologie, Bd. I/3, Teil A.) Berlin and New York: Springer, 1961.

Requests for reprints to: Dr P Luscieti, Istituto cantonale di patologia, 6600 Locarno, Switzerland. 